\title{
Utility of Point-of-Care Ultrasound in Children with Pulmonary Tuberculosis
}

\author{
Sabine Bélard, MD ${ }^{1,2,3,4}$, Charlotte C. Heuvelings, MD ${ }^{1,2}$, Ebrahim Banderker, FC Rad Diag \\ $(\mathrm{SA})^{5}$, Lindy Bateman, $\mathrm{MBCh}^{1}$, Tom Heller, $\mathrm{MD}^{6}$, Savvas Andronikou, $\mathrm{PhD}^{1,7}$, Lesley \\ Workman, MPH ${ }^{1}$, Martin P. Grobusch, FRCP ${ }^{2}$, and Heather J. Zar, PhD ${ }^{1}$
}

${ }^{1}$ Department of Paediatrics and Child Health, Red Cross War Memorial Children's Hospital, and MRC Unit on Child \& Adolescent Health, University of Cape Town, Cape Town, South Africa ${ }^{2}$ Centre of Tropical Medicine and Travel Medicine, Division of Infectious Diseases, Department of Internal Medicine, Academic Medical Centre, University of Amsterdam, Amsterdam, The Netherlands ${ }^{3}$ Charité - Universitätsmedizin Berlin, corporate member of Freie Universität Berlin, Humboldt-Universität zu Berlin, and Berlin Institute of Health, Department of Pediatric Pneumology and Immunology, Berlin, Germany ${ }^{4}$ Berlin Institute of Health (BIH), 10178 Berlin, Germany ${ }^{5}$ Department of Pediatric Radiology, Red Cross War Memorial Children's Hospital, Cape Town, South Africa ${ }^{6}$ Lighthouse Clinic, Kamuzu Central Hospital, Lilongwe, Malawi ${ }^{7}$ University of Bristol, United Kingdom

\section{Abstract}

Background-Point-of-care ultrasound (POCUS) detects extra-pulmonary tuberculosis (EPTB) in HIV-infected adults but has not been evaluated in children despite their higher risk of EPTB. This study's aims were to investigate feasibility of POCUS for EPTB in children, frequency of POCUS findings suggestive of EPTB, and time to sonographic resolution of findings with treatment.

\begin{abstract}
Methods-This prospective South African cohort study enrolled children with suspected PTB. POCUS for pleural, pericardial or ascitic effusion, abdominal lymphadenopathy, or splenic or hepatic micro-abscesses was performed and repeated at 1,3 and 6 months of TB treatment. Prevalence of POCUS findings and their association with HIV-infection was investigated in children with confirmed PTB (microbiologically proven), unconfirmed PTB (clinically diagnosed), or unlikely TB (respiratory disease that improved during follow-up without TB treatment).
\end{abstract}

Results-Of 232 children [median age 37 months (IQR 18;74)], 39(17\%) were HIV-infected. Children with confirmed or unconfirmed PTB had a higher prevalence of POCUS findings than children with unlikely TB [18/58(31\%) and 36/119(30\%) versus 8/55(15\%), $\mathrm{p}=0.04$ and $\mathrm{p}=0.03$, respectively]. Pleural effusion [ $\mathrm{n}=30(13 \%)]$ or abdominal lymphadenopathy $[\mathrm{n}=28(12 \%)]$ were the most common findings; splenic micro-abscesses $[\mathrm{n}=12(5 \%)]$ were strongly associated with

Corresponding author: Sabine Bélard, Department of Pediatric Pneumology and Immunology, Charité-Universitätsmedizin Berlin, Augustenburger Platz 1, 13353 Berlin, Germany, Phone: +49 30450566 182, Fax: +49 30450566 931, sabine.belard@ charite.de. Conflict of Interests: The authors have no conflicts of interest relevant to this article to disclose. 
confirmed PTB. Children co-infected with HIV and TB were more likely than HIV-uninfected children with TB to have abdominal lymphadenopathy [37\% versus $10 \%, \mathrm{p}<0.001]$ or splenic micro-abscesses [23\% versus 3\%, $\mathrm{p}<0.001$ ]. Most ultrasound findings resolved by 3 months with appropriate TB treatment.

Conclusions-POCUS for EPTB in children with PTB is feasible. The high prevalence of findings suggests that POCUS can contribute to timely diagnosis of childhood TB and to monitoring treatment response.

\section{Keywords}

tuberculosis; ultrasound; children; extra-pulmonary; point-of-care

\section{Background}

Childhood pulmonary tuberculosis (PTB) is increasingly recognized to contribute to the global tuberculosis (TB) burden. Estimates of TB incidence and prevalence in children vary and have large uncertainty intervals, supporting the difficulty in establishing a diagnosis of TB in children [1]. Recently, advances in pediatric PTB including improved specimen collection, new molecular diagnostic techniques and better culture methods have strengthened diagnostic strategies. However, even with these advances up to $50 \%$ of children clinically diagnosed with PTB test negative for Mycobacterium tuberculosis [2-4].

Timely identification of childhood TB is critical as children are at risk for rapid progression and dissemination, especially if they are immunosuppressed, malnourished or young [5, 6]. Common manifestations of disseminated or extra-pulmonary TB (EPTB) in children are TB meningitis, miliary TB, or abdominal TB. Autopsy studies show high rates of PTB [7] and of EPTB $[8,9]$ in sub-Saharan Africa, underlining the concern that many cases remain undiagnosed [3,10-12]. The prevalence of concurrent EPTB and PTB in children has not been prospectively studied.

A point-of-care ultrasound (POCUS) protocol (focused assessment with sonography for HIV-associated TB, FASH) was developed to improve diagnosis of EPTB in HIV-infected adults $[13,14]$. POCUS is increasingly part of diagnostic algorithms in emergency department settings [15]. However, POCUS for TB has not been well studied in children. Case series suggest that abdominal TB diagnosed by ultrasound often complicates pediatric PTB $[16,17]$. The aims of this study were to investigate the feasibility of POCUS for detection of EPTB in children with PTB, to investigate the frequency of findings suggestive of EPTB, and to assess time to sonographic resolution of findings under TB treatment. Results of this study would thereby allow evaluating a potential role of POCUS within diagnostic algorithms for childhood TB (timely diagnosis and delineation of extent of TB disease) as well as a potential role of POCUS within monitoring treatment response.

\section{Patients and Methods}

This prospective study was conducted at Red Cross War Memorial Children's Hospital, Cape Town, South Africa, between June 2013 and July 2015. Children aged <13 years with 
suspected PTB who were enrolled in a cohort study evaluating novel TB diagnostics [3, 4, $18,19]$ underwent clinician-performed POCUS for detection of EPTB within five days of enrolment. POCUS was repeated at follow-up visits at 1,3 and 6 months if any enrolment sonographic feature suggested EPTB. Management for TB was the responsibility of the attending doctor who was informed of the results of the POCUS examination.

Ethical approval was obtained from the Research Ethics Committee of the Faculty of Health Sciences, University of Cape Town. Written, informed consent was obtained from a parent or legal guardian; verbal assent was obtained from children older than 7 years.

\section{Study Population, clinical and laboratory procedures}

Children were enrolled if they met the following inclusion criteria: cough, plus one or more of the following criteria: i) failure to thrive or loss of weight over the past 3 months; ii) a positive tuberculin skin test (TST, positive: $\geq 10 \mathrm{~mm}$ in HIV-uninfected and $\geq 5 \mathrm{~mm}$ in HIVinfected); iii) chest X-ray (CXR) suggestive of TB; iv) known household or close TB contact. Exclusion criteria were TB treatment or prophylaxis for more than 72 hours.

Diagnostic testing for TB comprised history-taking, physical examination, basic hematology and clinical chemistry, HIV-testing, CXR (anterior-posterior and lateral), TST, and two separate induced sputa specimens for Xpert MTB/RIF (Xpert, Cepheid, CA, USA) and liquid mycobacterial culture (BACTEC MGIT; Becton Dickinson, MD, USA). CXRs were interpreted based on a standardized case reporting form. The radiologist was blinded to clinical and ultrasound data. All patients returned for follow-up visits 1 and 3 months after enrolment. Patients started on TB treatment had an additional follow-up visit at 6 months.

Patients were categorized into confirmed PTB (microbiologically confirmed i.e. either culture or Xpert positive for M. tuberculosis), unconfirmed PTB (clinical diagnosis and TB treatment without microbiological confirmation), or unlikely $T B$ (respiratory disease, but TB not microbiologically confirmed or clinically diagnosed and improved during follow-up without TB treatment) [20]. TB treatment was at the discretion of the treating doctor. Patients not started on TB treatment and lost to follow-up were excluded from analysis, as they could not be assigned a TB category.

\section{Ultrasound Assessment}

The ultrasound examination was carried out as a point-of-care test, at the bedside by a clinician with 5 years of clinical experience but no prior ultrasound experience who underwent a 4-day training period by an ultrasound specialist. Ultrasound was performed with a portable gray scale ultrasound machine (Mindray DP-10; Mindray, Shenzhen, China) using a convex and a linear probe (frequency ranges $2-5 \mathrm{MHz}$ and $5-10 \mathrm{MHz}$, respectively). Children were unsedated and scanned in supine position. The applied POCUS protocol (FASH) [13] with probe positions and sonographic definitions is shown in Supplemental Digital Content 1 (Table). In summary, children were sonographically examined for pericardial effusion, pleural effusion, ascites, abdominal lymphadenopathy (lymph nodes $\geq$ $10 \mathrm{~mm}$ short axis) as well as hepatic micro-abscesses. 
Representative sonographic pictures with pre-defined landmarks of the following positions were saved electronically: subcostal heart, pancreas, oblique porta hepatis, mesentery, right and left liver lobes, right and left costo-phrenic angles, Pouch of Morrison, sub-splenic area, splenic hilum, spleen, and transverse and longitudinal bladder; Supplemental Digital Content 1 (Table). Additional clips were saved if the examiner considered them helpful for interpretation. POCUS was interpreted by the examiner and separately, for the first half of the cohort, by a senior pediatric radiologist blinded to the examiner's interpretation and clinical data, according to a standardized reporting sheet. In case of discrepant interpretation of any finding between examiner and radiologist, a third ultrasound specialist blinded to the others' interpretation reviewed the findings; diagnosis was based on majority opinion.

The examiner documented the patient's acceptability of POCUS as good (patient was cooperative and remained calm), moderate (patient was crying but remained calm), poor (patient was crying and moving) or no compliance (POCUS could not be performed due to patient's non-compliance). Visualization conditions in the upper abdomen were documented as satisfactory (target landmarks entirely and clearly visible), partly obscured (target landmarks not entirely or not clearly visible) or obscured (target landmarks not identifiable).

\section{Data Analysis}

Data were extracted from the study database and imported into the statistical software SPSS (IBM Corp. Released 2013. IBM SPSS Statistics for Windows, Version 22.0. Armonk, NY).

Descriptive statistics were used to characterize the study population; normally distributed continuous data were summarized by mean and standard deviations (SD), non-normally distributed continuous data by median and interquartile range (IQR). Categorical data were summarized as number and proportion. Statistical tests included chi-square test (adjusted if number less than 5), Kruskal-Wallis comparison of medians and multivariate logistic regression. The multivariate logistic regression model was increased by adding each covariate separately. The best model was selected using the lowest Akaike's information criteria (AIC) and the likelihood ratio test $\mathrm{p}$ value was used to determine if this was a statistically significant result. Statistical tests were two-sided at $a=0.05$. Inter-reader agreement was determined using Cohen's kappa coefficient.

WHO Child Growth Standards were used to calculate weight-for-age and height-for-age zscores, a weight-for-age z-score of $\leq-2$ was classified as under-weight-for-age.

\section{Results \\ Demographics}

Of 237 children enrolled 4 children were excluded from analysis due to lost to follow-up and inability to assign TB category. Of 233 children with suspected PTB who had POCUS 58 (25\%) had confirmed PTB, 120 (51\%) had unconfirmed PTB, and 55 (24\%) had unlikely TB; Table 1. Thirty-nine (17\%) children were HIV-infected, 188 (81\%) were HIVuninfected, and the HIV status of $6(2 \%)$ children was unknown. The median age was 37 months (IQR 18;71); 82 (35\%) were aged less than two years; 110 (47\%) were female. Forty-five (22\%) children were classified as under-weight-for-age. Hepatomegaly or a 
distended or tender abdomen was documented in $48(21 \%)$ and $25(11 \%)$ children, respectively; abdominal signs and respiratory signs (indicative of pleural effusion) were only present in a minority; Table 1.

\section{Feasibility of point-of-care ultrasound}

Compliance with POCUS was rated good in 151/231 (65\%), moderate in 35/231 (15\%) and poor in 44/231 (19\%) children. In a single child (HIV-uninfected, unconfirmed PTB) POCUS could not be performed due to non-compliance, and for two children assessment of compliance was missing. Older age was significantly associated with better compliance; median (IQR) age in those with good compliance was $52(28 ; 97)$ months versus $16(12 ; 21)$ months in children with poor compliance $(\mathrm{p}<0.001)$.

The ultrasound examiner rated visualization of the upper abdomen as satisfactory in 126/229 (55\%) children, as partly obscured in 81/229 (35\%) children and as obscured in 22/229 $(10 \%)$ children; data on visualization was missing for three children. The radiologist rated visualization as satisfactory in 101/109 (93\%) children and partly obscured in 8/109 (7\%) children. Better visualization was significantly associated with older age; median (IQR) age in months in children rated with satisfactory versus obscured visualization was $49(24 ; 98)$ vs. $22(14 ; 37)$, respectively, $\mathrm{p}=0.003$. Overall, $5.7 \%$ of POCUS views were non-evaluable (due to obscuring bowel gas, empty bladder, or poor compliance); in decreasing frequency the five most common non-evaluable views pertained to assessment of mesenteric lymphadenopathy, epigastric lymphadenopathy, ascites, splenic hilum lymphadenopathy, and focal splenic lesion. There was high inter-observer agreement (kappa coefficient 0.80 ) for POCUS findings between the examiner and the radiologist; a third reading to resolve disagreement between these was required in only $1.9 \%$ of reviewed scans.

\section{Point-of-care ultrasound findings at presentation}

Children with confirmed or unconfirmed PTB had a higher rate of positive POCUS than children with unlikely TB [18/58 (31\%) and 36/119 (30\%) versus $8 / 55(15 \%), \mathrm{p}=0.037$ and $\mathrm{p}=0.030$, respectively]; Table 2 . There was a similar prevalence of positive POCUS in children with confirmed and unconfirmed PTB [18/58 (31\%) versus 36/119 (30\%), $\mathrm{p}=0.9$ ]. In 14 (8\%) children with confirmed or unconfirmed TB, more than one POCUS finding was observed.

Pleural effusion was the most common ( $\mathrm{n}=30,13 \%)$ POCUS finding. Pleural effusion by means of CXR was reported in 37 (16\%) children; for 24 children, pleural effusion was reported by both POCUS and CXR examination.

Abdominal lymphadenopathy $(\mathrm{n}=28,12 \%)$ was the second most common sonographic finding; focal splenic lesions $(n=12,5 \%)$ were strongly associated with confirmed PTB; Table 2.

In children with unlikely TB, pericardial effusion, focal splenic or hepatic lesions were not identified. Three children with unlikely TB also had abdominal lymphadenopathy. One child was diagnosed with hepatic and pulmonary cystic echinococcosis by means of POCUS. In this case, abdominal lymphadenopathy was probably due to hepatic cystic echinococcosis 
(abdominal lymphadenopathy resolved at 3 months of follow-up after albendazole treatment without TB treatment). One child was diagnosed with hepatitis A and one child was newly diagnosed with HIV infection, but TB was unlikely.

In children with confirmed or unconfirmed PTB, a positive POCUS was not associated with HIV-infection [12/30 (40\%) versus 42/147 (29\%), $\mathrm{p}=0.224]$, Table 3. However, amongst children with PTB, HIV-infection was more strongly associated with abdominal lymphadenopathy [11/30 (37\%) versus $14 / 147(10 \%)$, $\mathrm{p}<0.001]$ or focal splenic lesions [7/30 (23\%) versus 5/147 (3\%), p<0.001]; Table 3. In HIV-infected children the only ultrasound finding associated with confirmed PTB was focal splenic lesions (5/12 (42\%) confirmed PTB vs. 0/9 (0\%) unlikely TB, $\mathrm{p}=0.04)$.

Children with a positive POCUS were about 2.6 times more likely than children with a negative POCUS to have confirmed PTB rather than unlikely TB (RR (relative risk) 2.6; 95\% CI 1.1-6.7). Similarly those with a positive POCUS finding were 2.5 times more likely to have unconfirmed TB rather than unlikely TB (RR 2.5; 95\% CI 1.2-2.5). The possible effect of age less than two years, under-weight-for-age, and HIV status on the outcome of positive POCUS in all three TB categories was further explored by multinomial logistic regression, using children with unlikely TB as the reference category. After adjusting for the effect of these covariates, children with a positive POCUS were 2.8 times more likely than children with a negative POCUS to have confirmed TB rather than unlikely TB (RR 2.83; 95\% CI 0.94-8.53), although this is not statistically significant at the 0.05 level. Children with a positive POCUS were 2.7 times more likely than children with a negative POCUS to have unconfirmed TB rather than unlikely TB (RR 2.7; 95\% CI 1.01-7.20); Supplemental Digital Content 2 (Table).

\section{Evolution of point-of-care ultrasound findings with treatment for TB}

TB treatment comprised three drugs (rifampicin, isoniazid, pyrazinamide) in 51\% (90/177) children and four drugs (rifampicin, isoniazid, pyrazinamide and ethambutol or ethionamide) in $46 \%$ (82/177) children; $3 \%$ (5/177) children were treated with more than four drugs. Children with a positive POCUS were more likely to receive four or more drugs than children with a negative POCUS [37/54 (69\%) versus 50/123 (41\%), p=0.001]. Thirty-six (22\%) children needed TB treatment for more than six months but prolonged treatment was not associated with a positive POCUS [14/51 (28\%) versus 22/114 (19\%), $\mathrm{p}=0.24]$ at enrolment.

Follow-up POCUS data was obtained for 49 (91\%) of 54 children with confirmed or unconfirmed PTB and a positive POCUS at enrolment ( $n=46, n=39$, and $n=40$ children had sonographic follow up at 1,3, and 6 months, respectively); POCUS findings at 1, 3 and 6 months are presented in Table 4.

After 3 months of TB treatment POCUS findings had resolved in 69\% (27/39) of children. In 11/12 children with a POCUS still positive after 3 months of TB treatment, reasons for inadequate treatment response could be identified: five children had defaulted or incorrectly taken TB treatment, two children had not taken antiretroviral therapy (ART) as indicated, one child had multi-drug resistant TB, one child had developed immune reconstitution 
inflammatory syndrome (IRIS), one child was admitted to the intensive care unit (ICU) for severe disease and one child had non-tuberculous mycobacterial disease diagnosed on culture.

After 6 months of TB treatment, 33/40 (83\%) children had resolution of ultrasound findings while 7 children still had at least one POCUS finding. Of the 7, one child's TB medication was under-dosed, one child's TB medication was incomplete, one child had IRIS, one child was non-compliant with ART, and one child had been in ICU for 3 months due to complications of HIV/TB co-infection.

\section{Discussion}

This is the first study evaluating POCUS for identification of features of EPTB in children with suspected PTB. A third of children with PTB had sonographic features compatible with concurrent EPTB; this occurred in HIV-infected and uninfected children. Pleural effusion, followed by abdominal lymphadenopathy, were the most common ultrasound findings but splenic micro-abscesses were most strongly associated with PTB. While POCUS detected an additional six patients with pleural effusions, some cases were diagnosed on CXR but not on POCUS. POCUS may be more sensitive than CXR in detecting small amounts of fluid [2123] but there may be diagnostic value in performing both tests as POCUS only assesses the presence of pleural effusion in the costophrenic angles. In six of 13 patients with pleural effusion reported by CXR only, POCUS detected lung consolidation. Ultrasound has previously been reported to better distinguish consolidation from effusion than CXR [24]. Whether the pleural effusions detected on CXR are true effusions is not possible to confirm without a gold standard diagnostic such as CT chest. However, POCUS can provide additional information like the presence of loculations and guide placement of an intercostal chest drain.

The high prevalence of concurrent features of EPTB is indicative of the vulnerability of children to develop disseminated TB. In contrast, sonographic signs of extra-pulmonary involvement were less common in children other respiratory diseases and specific findings such as splenic micro-abscesses did not occur. Furthermore, there was resolution of ultrasound findings in most children with PTB by three months of treatment, suggesting that ultrasound may be a useful tool for monitoring of TB treatment response in children.

HIV-infection was not associated with an overall higher prevalence of positive POCUS. Previous surveillance studies reporting an increased risk of EPTB in HIV-infected children may be biased by HIV-infected children being more readily investigated for EPTB because of the perceived risk[25]. This study indicates that HIV-uninfected children are also at risk for EPTB, and underscores the need to evaluate all children with PTB for extra-pulmonary features. Furthermore, age was not associated with a positive POCUS; however, our cohort was young so these findings may not be generalizable to older children or adolescents. In most children, ultrasound findings resolved after 3 months of TB treatment; however, persistent changes 3 or 6 months after TB treatment initiation were related to incorrect TB or HIV treatment, non-compliance, or IRIS in most cases. Comparable temporal resolution of POCUS findings and association of persistent changes with TB treatment complications 
have also recently been reported for adults [26]. Sonographic follow-up after 3 months of TB treatment may therefore allow more reliable assessment of treatment response beyond clinical parameters.

Children with confirmed or unconfirmed PTB were more likely to have a positive POCUS than children with other respiratory disease but different ultrasound findings were associated with the diagnosis of either TB or of HIV-associated TB. Focal splenic lesions or abdominal lymphadenopathy were more common in HIV-infected children with PTB. Pleural effusion, the most common ultrasound finding, pericardial effusion, focal liver lesions, or ascites were not associated with TB. Therefore, in using ultrasound in children, interpretation of individual findings must be made critically. Although focal splenic lesions or abdominal lymphadenopathy in the clinical and epidemiologic context are more likely diagnostic of TB $[27,28]$, abdominal lymphadenopathy is a frequent observation in children not necessarily due to TB [29-31]. Differential diagnoses should also be considered in the presence of pericardial, pleural or ascitic effusions or with focal liver lesions. A comprehensive POCUS examination including thoracic and liver scanning nonetheless appears helpful in establishing a diagnosis of TB. Firstly, this procedure is relatively easily and quickly performed, with high reliability and without any radiation. Secondly, findings provide clinical information that may translate into further diagnostic or therapeutic interventions. For example pleural effusion can be drained for further diagnostic examinations or clinical relief and liver findings can establish a differential diagnosis (such as hydatid disease as occurred in this cohort). Thirdly, follow-up assessment of treatment response can be effectively done.

A limitation of the study is inclusion of hospitalized children who are likely to have more severe disease. The results may therefore not be generalizable to children with less severe disease; further study of ultrasound for TB in ambulatory children is needed. Sonographic findings suggestive of EPTB were not confirmed microbiologically as invasive specimen collection for this purpose would not have been justified clinically or ethically. However, sonographic resolution of ultrasound findings with TB treatment provides additional evidence that these were due to $M$. tuberculosis. While the sample size of children with confirmed and unconfirmed TB was large, analysis by HIV status was limited by the relatively small numbers of HIV-infected children with PTB. This reflects the strong mother to child HIV prevention program with substantial reduction in the number of HIV-infected children globally [32]. Future studies should include several POCUS operators trained according to a standardized POCUS curriculum and performing POCUS on the same patients, to improve generalizability of POCUS feasibility in children and assess for interoperator agreement; feasibility of short course training and high agreement rate between FASH trainees and their reviewer has previously been reported for FASH in adults [33].

The study has several strengths. It is the first study to investigate clinician-performed POCUS for childhood TB and to prospectively assess the prevalence of concurrent PTB and EPTB. Performance of the study in a clinical research setting where optimal TB diagnostics were available and careful follow-up allowed for clear case definitions. Review of ultrasound scans by a senior pediatric radiologist with high inter-reader agreement supports the quality and interpretation of sonographic data obtained. Finally, the use of a low-cost ultrasound 
device prospectively demonstrates that this is feasible as a suitable, affordable point-of-care diagnostic tool.

\section{Supplementary Material}

Refer to Web version on PubMed Central for supplementary material.

\section{Acknowledgments}

We are grateful to all study participants and their guardians as well as all personnel in the hospital and research unit who contributed to this study.

\section{Source of Funding}

NIH RO1 HD058971, MRC South Africa, NRF South Africa. SB and CCH were funded by a Marie Curie People grant and SB is currently participant in the BIH-Charité Clinician Scientist Program funded by Charité Universitätsmedizin Berlin and the Berlin Institute of Health. HZ is funded by the MRC South Africa.

\section{References}

1. World Health Organization. Global tuberculosis report 2016. 2016.

2. Nicol MP, et al. Accuracy of the Xpert MTB/RIF test for the diagnosis of pulmonary tuberculosis in children admitted to hospital in Cape Town, South Africa: a descriptive study. Lancet Infect Dis. 2011; 11(11):819-24. [PubMed: 21764384]

3. Zar HJ, et al. Rapid diagnosis of pulmonary tuberculosis in African children in a primary care setting by use of Xpert MTB/RIF on respiratory specimens: a prospective study. Lancet Glob Health. 2013; 1(2):e97-104. [PubMed: 25104164]

4. Zar HJ, et al. Rapid molecular diagnosis of pulmonary tuberculosis in children using nasopharyngeal specimens. Clin Infect Dis. 2012; 55(8):1088-95. [PubMed: 22752518]

5. Seddon JA, Shingadia D. Epidemiology and disease burden of tuberculosis in children: a global perspective. Infect Drug Resist. 2014; 7:153-65. [PubMed: 24971023]

6. Newton SM, et al. Paediatric tuberculosis. Lancet Infect Dis. 2008; 8(8):498-510. [PubMed: 18652996]

7. Bates M, et al. Burden of respiratory tract infections at post mortem in Zambian children. BMC Med. 2016; 14:99. [PubMed: 27363601]

8. Gupta RK, et al. Prevalence of tuberculosis in post-mortem studies of HIV-infected adults and children in resource-limited settings: a systematic review and meta-analysis. Aids. 2015; 29(15): 1987-2002. [PubMed: 26266773]

9. Bates M, et al. Burden of tuberculosis at post mortem in inpatients at a tertiary referral centre in subSaharan Africa: a prospective descriptive autopsy study. Lancet Infect Dis. 2015; 15(5):544-51. [PubMed: 25765217]

10. Connell TG, Zar HJ, Nicol MP. Advances in the diagnosis of pulmonary tuberculosis in HIVinfected and HIV-uninfected children. J Infect Dis. 2011; 204(Suppl 4):S1151-8. [PubMed: 21996697]

11. Graham SM, et al. Evaluation of tuberculosis diagnostics in children: 1. Proposed clinical case definitions for classification of intrathoracic tuberculosis disease. Consensus from an expert panel. J Infect Dis. 2012; 205(Suppl 2):S199-208. [PubMed: 22448023]

12. Planting NS, et al. Safety and efficacy of induced sputum in young children hospitalised with suspected pulmonary tuberculosis. Int J Tuberc Lung Dis. 2014; 18(1):8-12. [PubMed: 24365546]

13. Heller T, et al. Focused assessment with sonography for HIV-associated tuberculosis (FASH): a short protocol and a pictorial review. Crit Ultrasound J. 2012; 4(1):21. [PubMed: 23171481]

14. Belard S, et al. Point-of-Care Ultrasound Assessment of Tropical Infectious Diseases--A Review of Applications and Perspectives. Am J Trop Med Hyg. 2015; 94(1):8-21. [PubMed: 26416111] 
15. van Hoving DJ, et al. Adequacy of the emergency point-of-care ultrasound core curriculum for the local burden of disease in South Africa. Emerg Med J. 2013; 30(4):312-5. [PubMed: 22593261]

16. Scheepers $S$, et al. Abdominal lymphadenopathy in children with tuberculosis presenting with respiratory symptoms. Ultrasound. 2011; 19:134-139.

17. Belard S, et al. Sonographic findings of abdominal tuberculosis in children with pulmonary tuberculosis. Pediatr Infect Dis J. 2017 in press.

18. Nicol MP, et al. Urine lipoarabinomannan testing for diagnosis of pulmonary tuberculosis in children: a prospective study. Lancet Glob Health. 2014; 2(5):e278-84. [PubMed: 24818083]

19. Nicol MP, et al. Xpert MTB/RIF testing of stool samples for the diagnosis of pulmonary tuberculosis in children. Clin Infect Dis. 2013; 57(3):e18-21. [PubMed: 23580738]

20. Graham SM, et al. Clinical Case Definitions for Classification of Intrathoracic Tuberculosis in Children: An Update. Clin Infect Dis. 2015; 61(Suppl 3):S179-87.

21. Emamian SA, et al. Accuracy of the diagnosis of pleural effusion on supine chest X-ray. European radiology. 1997; 7(1):57-60.

22. Röthlin MA, et al. Ultrasound in blunt abdominal and thoracic trauma. J Trauma. 1993; 34(4):48895. [PubMed: 8487332]

23. Blackmore CC, et al. Pleural fluid volume estimation: a chest radiograph prediction rule. Acad Radiol. 1996; 3(2):103-9. [PubMed: 8796649]

24. Yu CJ, et al. Ultrasound study in unilateral hemithorax opacification. Image comparison with computed tomography. Am Rev Respir Dis. 1993; 147(2):430-434. [PubMed: 8430970]

25. Hesseling AC, et al. High incidence of tuberculosis among HIV-infected infants: evidence from a South African population-based study highlights the need for improved tuberculosis control strategies. Clin Infect Dis. 2009; 48(1):108-14. [PubMed: 19049436]

26. Heller T, et al. Changes of FASH ultrasound findings in TB-HIV patients during anti-tuberculosis treatment. Int J Tuberc Lung Dis. 2014; 18(7):837-9. [PubMed: 24902561]

27. Malik A, Saxena NC. Ultrasound in abdominal tuberculosis. Abdom Imaging. 2003; 28(4):574-9. [PubMed: 14580103]

28. Heller T, et al. Abdominal tuberculosis: sonographic diagnosis and treatment response in HIVpositive adults in rural South Africa. Int J Infect Dis. 2010; 14(Suppl 3):e108-12. [PubMed: 20363170]

29. Vayner N, et al. Mesenteric lymphadenopathy in children examined by US for chronic and/or recurrent abdominal pain. Pediatr Radiol. 2003; 33(12):864-7. [PubMed: 13679999]

30. Sivit CJ, Newman KD, Chandra RS. Visualization of enlarged mesenteric lymph nodes at US examination. Clinical significance. Pediatr Radiol. 1993; 23(6):471-5. [PubMed: 8255656]

31. Karmazyn B, et al. Mesenteric lymph nodes in children: what is normal? Pediatr Radiol. 2005; 35(8):774-7. [PubMed: 15883829]

32. Evans C, Jones CE, Prendergast AJ. HIV-exposed, uninfected infants: new global challenge in the era of paediatric HIV elimination. Lancet Infect Dis. 2016; 16(6):e92-e107. [PubMed: 27049574]

33. Heller T, et al. Short course for focused assessment with sonography for human immunodeficiency virus/tuberculosis: preliminary results in a rural setting in South Africa with high prevalence of human immunodeficiency virus and tuberculosis. Am J Trop Med Hyg. 2010; 82(3):512-5. [PubMed: 20207884] 


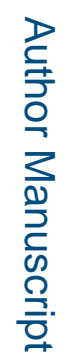

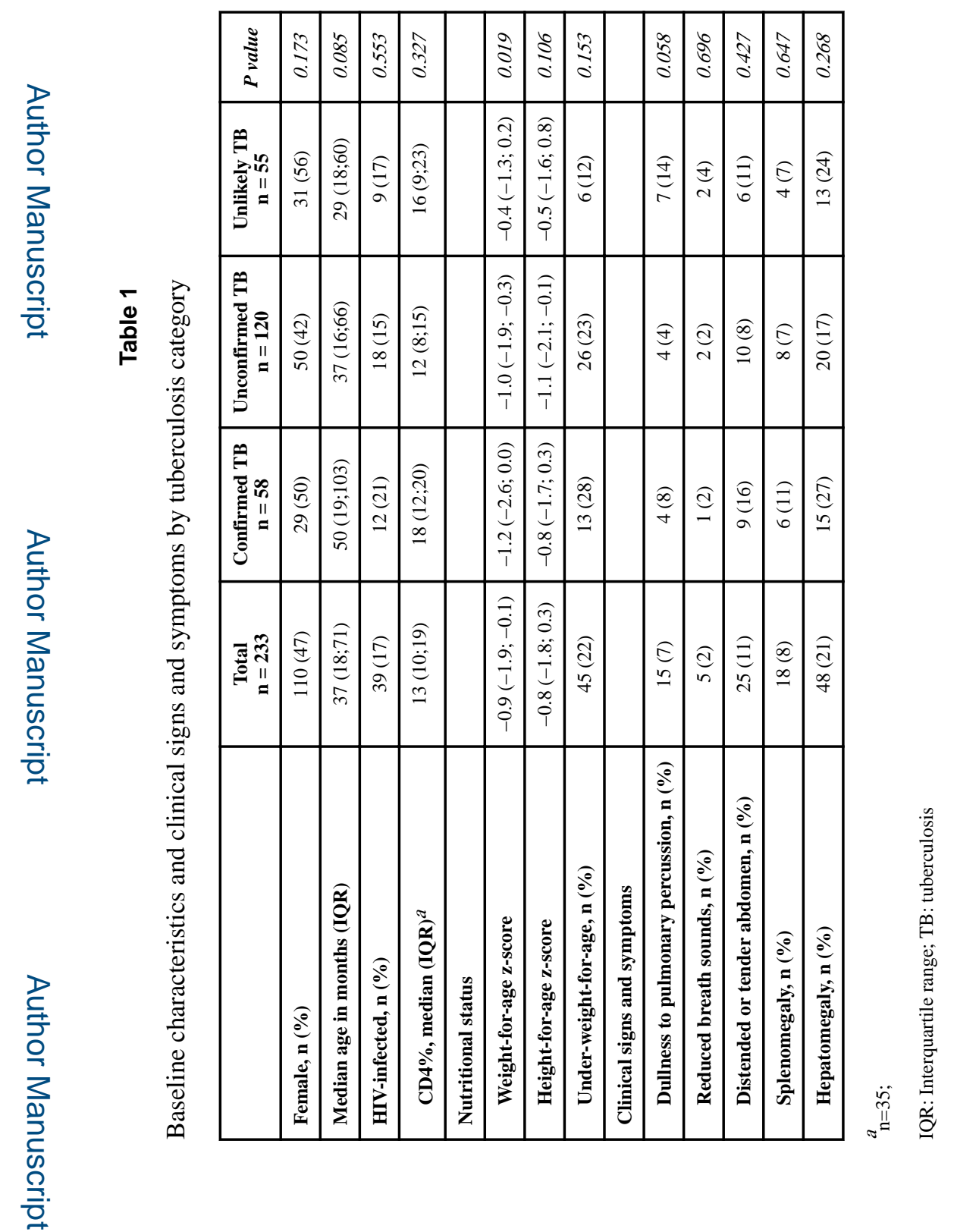

Pediatr Infect Dis J. Author manuscript; available in PMC 2019 July 01. 


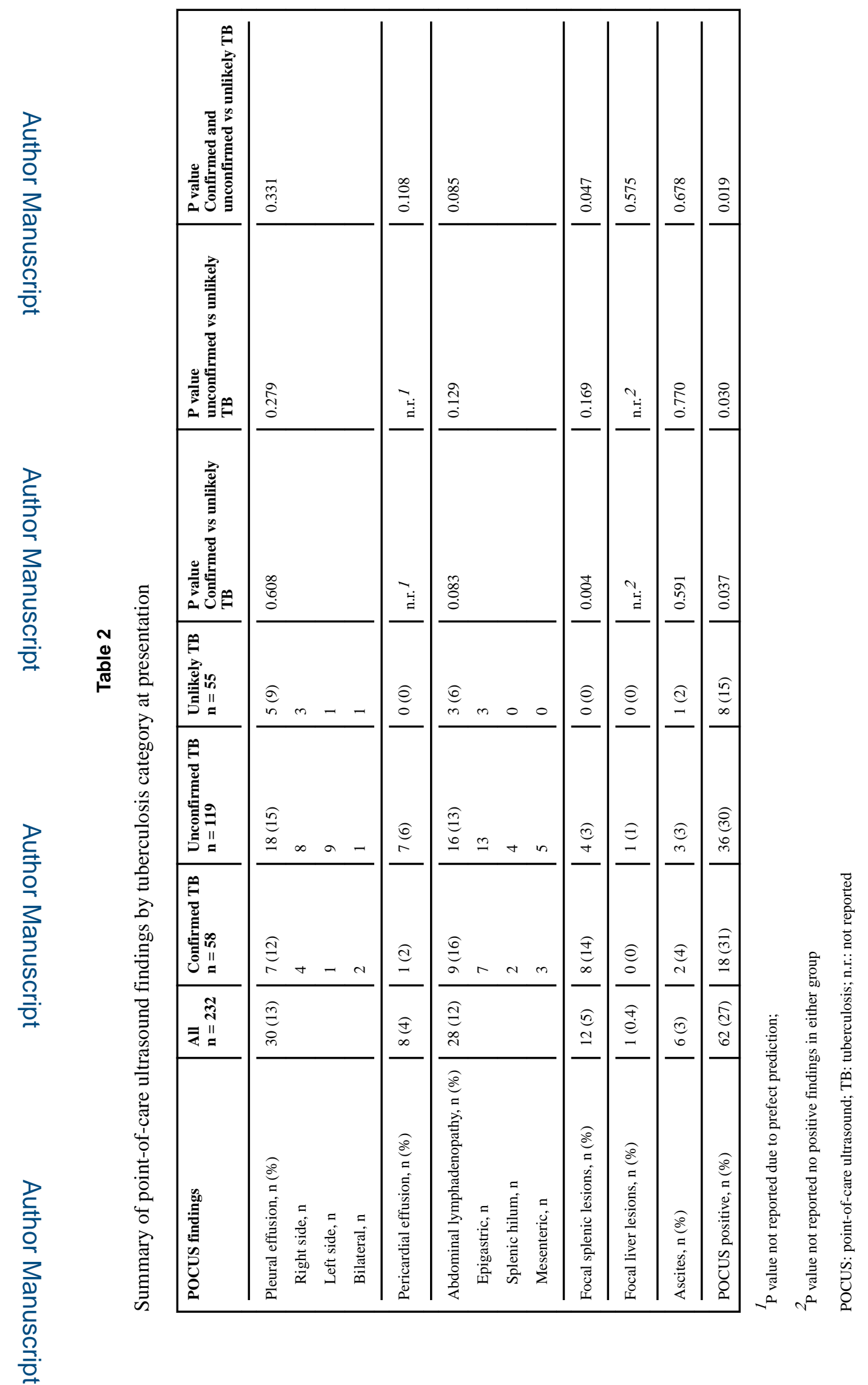

Pediatr Infect Dis J. Author manuscript; available in PMC 2019 July 01. 


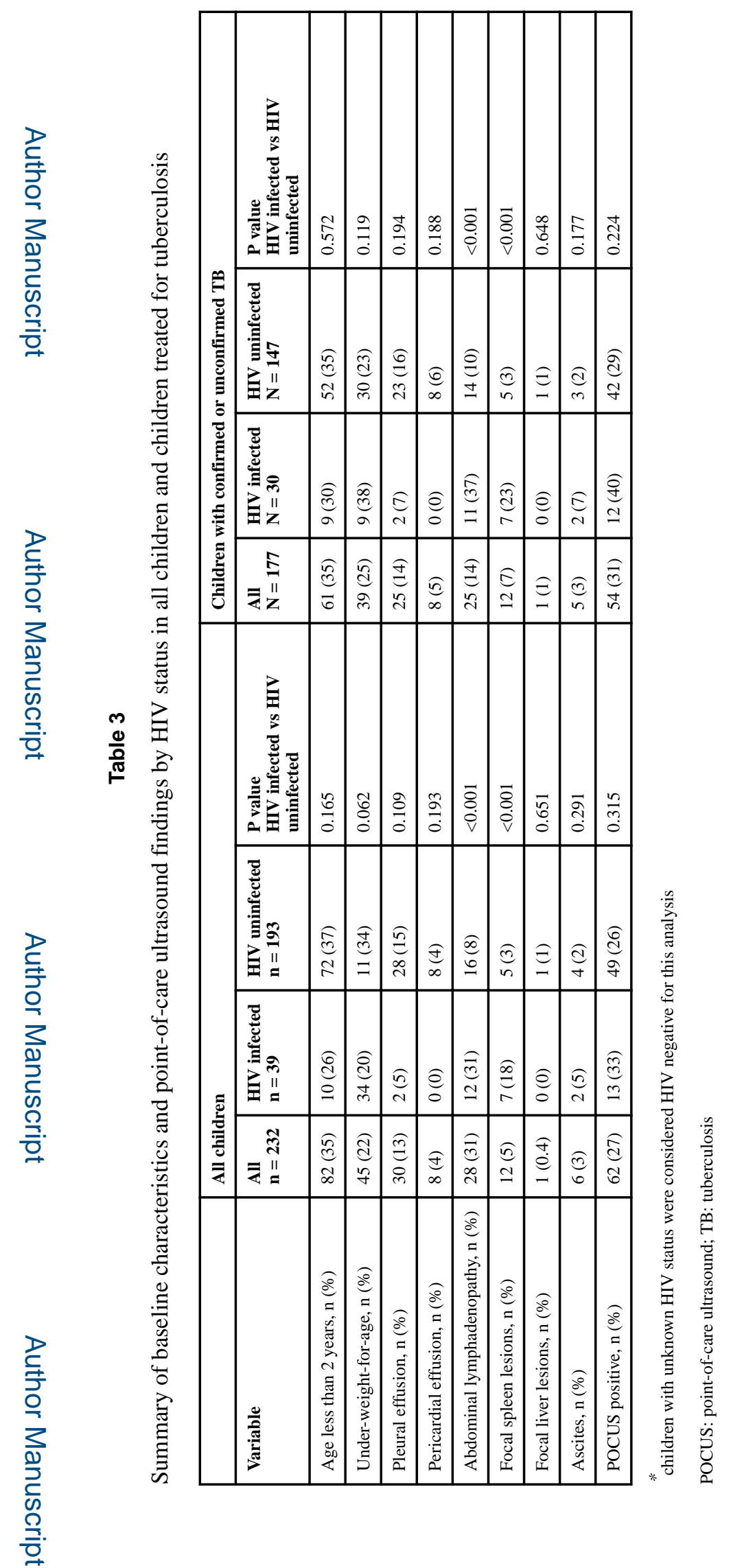

Pediatr Infect Dis J. Author manuscript; available in PMC 2019 July 01. 


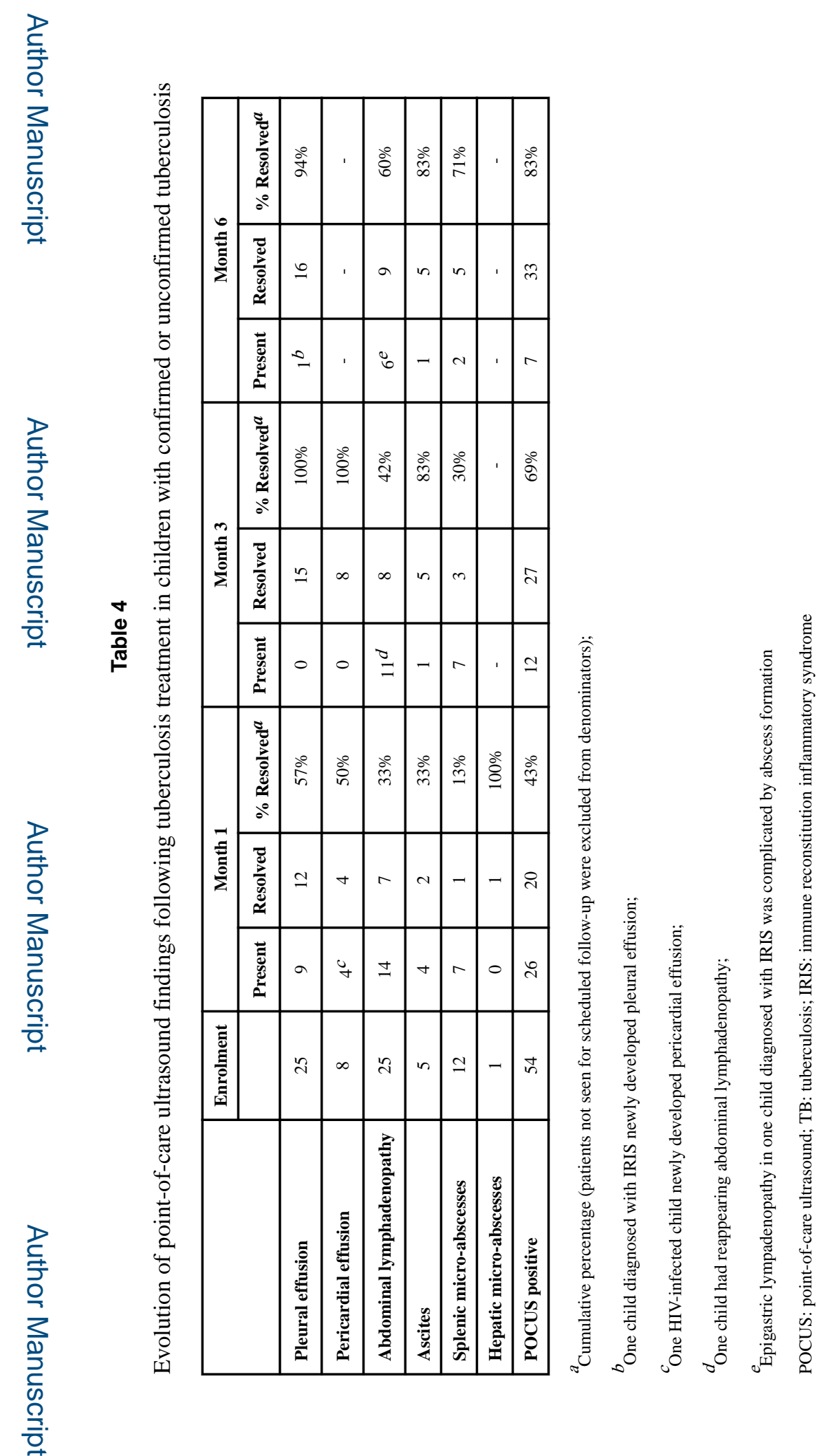

Pediatr Infect Dis J. Author manuscript; available in PMC 2019 July 01. 